http://dx.doi.org/10.12775/szhf.2018.004

\author{
Miroseaw ŻelazNy \\ Uniwersytet MikoŁaja Kopernika, Toruń, Polska \\ e-mail: zelazny@umk.pl
}

\title{
Kant und Jaspers ${ }^{*}$
}

Ich bin der Ansicht, dass die von Hannah Arendt vorgeschlagene Bezeichnung „Kant des zwanzigsten Jahrhunderts” sehr gut die Person und das Schaffen Jaspers bezeichnet. Mit großer Sicherheit war er kein Kantianist oder Neokantianist. Der Kantianismus wird im großen Masse zurecht mit dem Philosophieren, für das die Erkenntnistheorie vor allem im Bereich des spekulativen Verstandes am wichtigsten ist, assoziiert. Zum wichtigsten Ziel der Philosophie wurde bei dieser Interpretation das Bilden von Werkzeugen für die Bedürfnisse einer entsprechenden Entwicklung der Wissenschaft. Von den Wissenschaften schreibend, dachte Kant vor allem an die Mathematik und die Naturwissenschaft. Solch eine Interpretation dominierte im großen Masse das Denken in kantianischen Schulen. Ihre Vertreter werden hauptsächlich mit Personen assoziiert, die eine entsprechende Entwicklung der Wissenschaft gerne ermöglichen würden, indem sie diese Wissenschaften zu wahrlich exacten machen würden.

Ein durchschnittlicher Leser würde an dieser Stelle die Frage stellen, ob für die Wissenschaften und vor allem die exacten Wissenschaften bei ihrer Entwicklung überhaupt eine Philosophie nötig ist. Diese Frage kann positiv beantwortet werden, doch solch eine Antwort an sich erweist sich als verworren und schwer verständlich.

\footnotetext{
Referat wygłoszony 9 października 2017 roku, w Karl Jaspers-Gesellschaft w Oldenburgu.
} 
Die Neokantianisten betrieben natürlich auch eine eher humanistische Philosophie. Sie beschäftigten sich mit den Fragen aus dem Bereich der Ethik oder Ästhetik. Doch wieder schienen ihre Ausführungen in beiden Bereichen verwirrend und wirklichkeitsfremd zu sein. Es fehlte ihnen an einer klaren Präsentation, dank der die kantianistische Imperativ-Theorie bis heute außerordentlich nützlich für alle bleibt, die sich mit der Gesetzgebung beschäftigen, sei es der moralischen oder der erlassenen. Diese Klarheit der Ausführung trägt dazu bei, dass die kantianistische Imperativ-Theorie gleich wirksam angewandt werden kann und zwar sowohl indem man nach den persönlichen moralen Entscheidungen in belanglosen Angelegenheiten fragt, sowie auch nach den Wahlen im Bereich der internationalen Politik. Wenn es um die ästhetischen Werte geht, dann haben die Kantianisten und Neokantianisten keine zweite Kritik der Urteilskraft geschaffen. Ihre Werke, die sich hauptsächlich mit der Klassifizierung psychischer Prozesse beschäftigen, waren nicht in der Lage so fruchtbar auf die Künstler zu wirken, wie der Gedanke Kants z.B. auf Friedrich Schiller Einfluss hatte.

Jeglichen Proben der wissenschaftlichen Untermauerung s.g. gesellschaftlicher Wissenschaften würde Kant auch mit Misstrauen angehen, vor allem dann wenn wir als Wissenschaftsmuster die Naturwissenschaften nehmen. Der Autor der Kritik der reinen Vernunft war doch der Ansicht, dass man mithilfe von determinierenden Rechten erfolgreich die Phänomene der unbelebten Natur erklären kann, aber nicht die organischen Prozesse. Die Gesellschaft hingegen erkannte er entschieden als eine organische Struktur an, welche durch die Kausalität der Freiheit regiert wird.

Er könnte mit Sicherheit ideal vorhersehen, welchen Fehler Smith und Marks begannen haben, der die heutige Politik lähmt. Er beruht darauf, dass die gesellschaftlichen Prozesse als mechanische Prozesse behandelt werden, die angeblich beliebig anhand von kausal wirkenden Impulsen gesteuert werden können.

Die genannten Ursachen bewirkten, dass Jaspers, so wie Heidegger, eine Abneigung gegenüber neokantianischen Schulen verspürte. Wir sehen das anhand des Beispiels, das so dramatisch an den Streit mit Rickert in der $A u$ tobiographie erinnert. Jaspers Meinung nach hat die kantianische und neokantianische Tradition von den drei durch Kant formulierten philosophischen Hauptfragen: „Was kann ich wissen?", „was soll ich tun?", „was kann ich erwarten?" nur die erste ernst aufgenommen. ${ }^{1}$ In Bezug auf die zweite

${ }^{1}$ I. Kant, Logik, [in:] Gesammelten Werken, Akadmie-Ausgabe, Bd. IX, S. 25. 
Frage, welche unsere Handlungsweise betrifft, hat man sich hauptsächlich mit den Erwägungen beschäftigt, wie sie gestellt werden könnte. Man tat dies so erfolgreich, dass man im Allgemeinen die Titelfrage „was sollte ich eigentlich tun" nicht mehr wiederaufnahm. Die dritte Frage „was kann ich erwarten?" ist im philosophischen Sinne nicht mehr aufgenommen worden, man gab oftmals von vornherein fertige Antworten. Wir hörten also, dass wir ein gerechtigeres gesellschaftliches System, einen größeren gesellschaftlichen Reichtum u. ä. erwarten sollen. Doch auf die Fragen, welche ich zur Jugendzeit meinen Marxismus-Lehrern gestellt habe: „Was für einen Sinn hat das, dass der Proletarier sein Leben für den Kommunismus lässt, den er nie zu sehen bekommt, wobei er dafür seine Frau und seine Kinder hinterlassen muss?" habe ich keine Antwort erhalten. Das waren übrigens zu dieser Zeit im Osten Europas keine sicheren Fragen.

Die jungen Menschen, die ihren Kontakt mit der Philosophie beginnen, werden hingegen vor allem eben von der zweiten und dritten Kantianischen Frage berührt. Ich finde persönlich, dass in diesem Falle am wichtigsten eben die dritte Frage ist: was kann ich erwarten. Diese tritt in zahlreichen Dilemmas in Erscheinung, welche uns auf jedem Schritt quälen, und am häufigsten um so intensiver hervortreten, je näher wir den Tode sind.

Sie werden vor allem in dem Dilemma ausgedrückt: wieso bin ich zur Welt gekommen? Die Beobachtung einer unerhörten Präzision und Komplexität des Funktionierens von Naturphänomenen bewegt mich zur Schlussfolgerung, dass die Ganzheit der Natur durch irgendeine vernünftige Absicht regiert wird. Wieso jedoch die Realisierung jeglicher Ziele in der belebten Natur immer so dramatisch endet: der Tod eines Organismus, oftmals schmerzhaft und grausam? Wozu die ganze Präzision der Struktur und die Wirkung der Phänomene, wenn doch irgendwann das ganze Universum aufhören muss zu existieren? Wie soll in solch einer Situation der Weg zu Gott gesucht werden, von dem wir erwarten, dass der Gegensatz zwischen der Idee des Guten und der Idee der Natur aufgehoben wird, der außerhalb der Reichweite des Guten und Bösen existiert?

Eine ungeheure Anzahl an Philosophen meinte, dass bevor wir uns dieser Fragen annehmen, wir erst entsprechende Werkzeuge vorbereiten müssen, indem wir die erste Frage stellen: „was können wir wissen?“. So traten sie in den Bereich dieser Frage und kamen nicht mehr aus ihm heraus. Ein klassisches Beispiel wäre hier die Phänomenologie von Husserl, welche etwas verspricht, was man nach dem Beenden von erkenntnistheoretischen Erwägungen erzielen kann, indem man sich, Jaspers Meinung nach, utopische Ziele 
stellt. Ein anderes Beispiel könnte hier die Philosophie Hegels sein, dem es schien, dass er in seiner Logik alle Verwirrtheiten der Frage gelöst hat: „was kann ich wissen?" und dass er zu den zwei restlichen Fragen übergegangen ist. Der Effekt dieses Übergangs würde nämlich miserabel ausfallen. So wie Kierkegaard zurecht geschrieben hat, sind seine Antworten, in der Frage wo sich der Ort des persönlichen Glückes einer Einheit in dem System des absoluten Geistes befindet, sehr bescheiden.

Im Wesentlichen gelang es Hegel von der Frage „was können wir wissen?“ zu den zwei restlichen durch Kant gestellten Fragen nicht wirksam überzugehen. Die Ursache dafür gab Heidegger hervorragend in einem der Briefe an Arendt an: Hegel versuchte, indem er die Dialektik benutzte, clever das Problem zu umgehen, mit dem die Metaphysik nicht zurechtgekommen war.

Den Kern des Umgehens, dessen Autor in Wirklichkeit schon Fichte war, beschreibt Jaspers in vierten Kapitel des ersten Bandes seiner Philosophie, bei der Gelegenheit der Kritik des Idealismus.

Eins der wichtigen Probleme der Erkenntnistheorie ist der evidente Unterschied zwischen dem Gegenstand, der durch den äußeren Sinn des Daseins wahrgenommen wird, und der Vorstellung oder der Erinnerung des inneren Sinnes. Da jedoch jedes ,jetzt“ sofort zu einem „war“ wird, tritt die Versuchung in Erscheinung logisch zu erklären, dass der sogenannte äußere Sinn nur eine gewisse Phase der Aktivität des inneren Sinnes darstellt. Tatsächlich, so Fichte, ist dieser Sinn nur eine Täuschung, welche innerhalb der Triade erfolgt: Ich gleich Ich, Ich konfrontiert mit nicht-Ich, Ich kehrt zur Einheitlichkeit mit sich zurück. Für Kant ist solch eine Lösung des Problems der Grenze zwischen dem äußeren und inneren Sinn eine reine Scharlatanerie. Dies stellt einen Umgehungsversuch auf der Plattform einer unentsprechend angewandten Logik an einem Abgrund dar, die von metaphysischem Charakter ist. Mit großer Sicherheit würde er alle Versuche des Ersetzens der Metaphysik durch die Dialektik als Sophisma anerkennen. Da aber, wenn man hier Hume zitieren kann, auf einem gemalten Haken nur ein gemaltes Gewicht angebracht werden kann, so müssen alle Versuche der Antwort auf die Frage: „was sollte ich tun?" und „was kann ich erwarten?" bei einer idealistischen Lösung schwache Resultate erbringen. Kierkegaard schrieb, dass Hegel einen prächtigen Palast aufstellte, um neben ihm in einer Hundehütte zu wohnen.

Die beiden Hauptfragen der Philosophie, die zweite und dritte, können endlich gestellt werden, ohne dass man sich der erkenntnistheoretischen $\mathrm{Ba}$ sis annehmen muss. Wenn es um die Philosophie geht, so waren die Meister 
solch einer Herangehensweise: Boethius, Pascal, oder Kierkegaard. Man kann sich zu diesem Zweck auch der Literatur bedienen, um an die in dem zweiten Band der Philosophie enthaltenen hervorragenden Erwägungen in Bezug auf das Schaffen von Dostojewski und Shakespeare zu erinnern. Oftmals werden jedoch die Fragen, was sollten wir tun, und was können wir erwarten, von Schreibwütigen, von Berühmtheiten heimgesuchten Personen, Verkündern von angeblich offenbarten Wahrheiten aufgenommen. In der Sphäre des gesellschaftlichen Lebens werden uns die Antworten von Ideologen und Verkündern der Propaganda, wie den Nazis, aufgezwungen, die Jaspers über viele Jahre von der Nähe aus beobachten konnte.

Aus dieser Perspektive offenbart sich Kant Jaspers als der einzige Denker seiner Art. Er betrat das Innere der Frage „was können wir wissen?“, aber er blieb in ihr nicht stecken. Seine Ethik wurde durch die Erkenntnistheorie und die Klassifizierungen nicht so belastet, dass nicht viel von der Ethik selbst in ihr verblieb. Seine Philosophie des Gottes berief sich auf keine wissenschaftlichen Pseudodokumente, wie auch auf keine angeblich geoffenbarten Wahrheiten, welche in Wahrheit durch politische Methoden erzwungen wurden. Der Meinung Jaspers nach gelang solch ein Philosophieren nur Kant, nach der Ansicht von Hannah Arendt auch Jaspers.

Dies ergab sich aus der grundsätzlichen Tatsache: Jaspers behandelte, ähnlich wie Kant, die Idee der Einheit als eine Struktur, welche der Verstand der Natur auferlegt, aber er suchte sie nicht als eine Sache in sich selbst. Er verzichtete von vornherein auf die Versuche, irgendein einheitliches System zu bauen, von dessen Erreichen erst das Stellen weiterer philosophischer Fragen abhängig sein sollte. Indem er mit Heidegger über das Thema der in Sein und Zeit aufgenommenen Proben des Baus einer Ontologie diskutierte, stellte er sofort fest: das kann nicht gelingen.

Wenn wir nach der Ähnlichkeit zwischen der Philosophie von Kant und Jaspers im Sinne der Erkenntnis fragen, dann äußert sie sich, meiner Meinung nach, in einer eigenartigen Skepsis, welche man auf folgende Weise charakterisieren kann:

$\mathrm{Zu}$ allererst, führt unsere Erkenntnis zu zwei autonomischen Quellen: der sinnlichen Erkenntnis und dem Denken in Begriffen. In dem materiellen Sinne lassen sich die Quellen nicht zu einer einheitlichen Erkenntnis führen. Der Intellekt kann nicht sehen, die Sinne können nicht denken. Die Einheit beider Sphären ist ausschließlich von einem formellen und keinem materiellen Charakter. Sie entsteht für die Bedürfnisse des Erkenntnisprozesses. Sie ist keine Spiegelung irgendeiner einheitlichen Wirklichkeit in sich selbst. Der 
Verstand schöpft nicht aus den Rechten der Natur, sondern er diktiert sie der Natur. Das bedeutet aber nicht, dass er in der Lage ist, seine Rechte allem zu diktieren, was wir als Natur erfahren. Er verfügt zum Beispiel über keine Rechte, welche auf eine determinierende Weise das Phänomen des Lebens erklären würden. Kant meint, dass es nie einen Grashalm Newtons geben wird.

Zweitens: im Resultat der Möglichkeit des Bildens praktischer Philosophie können die beiden Quellen der Erkenntnis nicht bedingungslos von der Möglichkeit des Bildens einer idealen spekulativen Philosophie abhängig sein. An dieser Stelle verbindet sich die These Kants - „der bestirnte Himmel über mir und das moralische Gesetz in mir" ${ }^{\text {"2 }}$ - mit der These Kierkegaards: ginge alles auf der Welt vernünftig zu, dann würde nichts auf der Welt passieren. Wie für Kierkegaard das System Hegels auf einer Illusion des Erschaffens einer Philosophie fußte, welche eine Einheit der spekulativen und praktischen Philosophie bilden sollte, so betrifft Jaspers Meinung nach diese Illusion auch die Phänomenologie. Diese Kritik betrifft auch Sein und Zeit von Heidegger.

Drittens: es gibt keine einheitliche materielle Wirklichkeit. Die Einheit der Wirklichkeit ist ausschließlich transzendentalen Charakters, und nicht transzendent. Das ist eine Einheit, welche durch die Strukturen des reinen Intellektes gebildet wird, und nicht durch die Einheit der Natur in sich selbst. Sie reicht nur dorthin, wohin auch der Intellekt reicht. Der Intellekt hingegen umfasst nicht einmal alle Phänomene, die der Mensch wahrnimmt.

Die wunderschöne Allegorie über den mit Sternen besetzten Himmel und das moralische Gesetz bedeutet auf den ersten Blick eine Inkongruenz der Welt des natürlichen Determinismus mit der Welt der Freiheit. Aber auch in der außermenschlichen Natur selbst kommt das Phänomen zu Tage, dass es nicht zu der bestimmenden Kausalität gehört, sondern zur Kausalität der Freiheit. Das ist das Phänomen des Lebens. Den Kern der Kantianischen Vision der Wirklichkeit würde also die These ausdrücken, welche aus zwei bekannten Phrasen besteht. Die erste lautet „der bestirnte Himmel über mir und das moralische Gesetz in mir", und die zweite hingegen „es wird niemals den Grashalm Newtons geben“. Die Kausalität der Freiheit überschreitet auf ihre Weise die Grenze der Aufteilung in eine natürliche und eine menschliche Welt, obwohl sie in diesen beiden Welten durch uns unterschiedlich empfunden wird.

${ }^{2}$ I. Kant, Kritik der praktischen Vernunft, [in:] Gesammelten Werken, Akadmie-Ausgabe, Bd. V, S. 161. 
Jaspers nimmt eine grundsätzliche Teilung der Wirklichkeit in eine Wirklichkeit der Welt und des Geistes vor. Das ist eine Aufteilung, welche mit dem Buchstaben der Philosophie von Kant übereinstimmt, obwohl dieser keinen direkten Begriff des Geistes als einer Bezeichnung der ganzen menschlichen Wirklichkeit einführt.

Jaspers teilt, indem er Kant folgt, die Wirklichkeit der Welt in die Wirklichkeit der unbelebten und der belebten Natur auf. Die erste von ihnen ist durch Kant der bestimmenden, die zweite der reflexiven Urteilskraft untergeordnet worden. Jaspers benutzt nicht genau diese Begriffe, aber das Prinzip der Aufteilung ist für ihn genau das gleiche, wie für Kant.

Die Wirklichkeit der belebten Natur hat ihre eigenen bestimmenden Rechte der Natur. Für Kant waren das die Rechte der Newton-Mechanik, für Jaspers die ständig entdeckenden Physikrechte und die sich im Laufe des Fortschrittes ersetzenden Erkenntnisse der Physikrechte und der mit ihnen verbundenen exakten Wissenschaften.

Die These der Wirklichkeit der belebten Natur wird niemals zugunsten Newtons ausfallen. Ein Grashalm als These bewahrt für Jaspers an Aktualität, dass es niemals möglich sein wird, das Phänomen des Lebens durch die Anwendung von Herrschern der unbelebten Natur zu erklären.

Natürlich gab es die positivistischen fordernden Theorien, dass das Leben spontan aus der unbelebten Natur heraus entstanden sei, schon immer, und es wird sie immer geben. Jaspers kritisiert sie in dem schon genannten vierten Kapitel des ersten Philosophiebandes. Er erkennt sie als positivistisch an und schreibt kurz zu ihnen: „Die Voraussetzung der Erkennbarkeit von allem lüßt vorgrafend das Erkannte zum Weltbild sich runden; es ist, als ob alles nicht begreiflich, sondern im Prinzip schon Begriffen wäre"3.

Das ist eine These, welche den Philosophen und Wissenschaftlern sehr gut bekannt ist. Hume fasste sie in seine bekannte These: die Aufdringlichkeit einer Frage kann keine Antwort aufzwängen.

Obwohl also die These, dass das Leben als spontaner Effekt einer unbelebten Materie entstanden ist, als selbstverständlich erscheint, und ihre Infragestellung in gewissen Kreisen als eine Häresie angesehen wird, so ändert sie nicht die grundsätzliche Tatsache: es ist uns nichts darüber bekannt, dass sie jemals jemand experimentell bestätigt hat. Alle Informationen dazu, dass es jemandem gelungen ist, enden sehr schnell mit einer Kompromittierung.

${ }^{3}$ K. Jaspers, Philosophie, Berlin-Göttingen-Heidelberg 1948, S. 182. 
In diesem Punkt ist Jaspers ein treuer Schüler Kants. Beide meinen, dass bei allen in der Geschichte beobachteten Fällen, das Leben immer einen Effekt eines anderen Lebens darstellte, das es ins Leben rief.

Natürlich verbinden die Phänomene der unbelebten und belebten Natur evidente Korrelate, aber diese Korrelate sind doch schon in ihrer Natur kein Beweis dafür, dass zwei Phänomene durch eine enge resultierende Beziehung miteinander verbunden sind. Der Übergang von der Wirklichkeit der unbelebten Natur zur Wirklichkeit der belebten Natur wird niemals ein tatsächlicher Übergang, aber immer ein Übersprung sein. Jeder Versuch der Negation dieser Wahrheit müsste sich auf wissenschaftliche Errungenschaften beziehen, welche noch nicht erlangt worden sind, obwohl angeblich „alles auf sie hinweist".

In Wahrheit jedoch unterscheidet die Phänomene der unbelebten Natur von den Phänomenen der belebten Natur die Tatsache, dass die einen der bestimmenden Kausalität unterstehen, und die anderen der Kausalität der Freiheit. Der Übergang von der einen zu der anderen stellt einen grundsätzlichen qualitativen Sprung dar. Uns sind keine Tatsachen bekannt, dass die Gegenstände der unbelebten Natur auf einmal etwas möchten, oder eine spontane Initiative des Überlebens in eben dieser Form an den Tag bringen (obwohl es auch hier gewisse Korrelate und Analogien gibt). Die Phänomene der unbelebten Natur streben nach keinen Zielen, welche durch die Kausalität der Freiheit geweckt werden. Sie können nur zu gewissen Zuständen gelenkt werden, welche durch den außenstehenden Beobachter mit Zielen assoziiert werden. Dann jedoch werden diese Ziele nicht solche jener Phänomene sein, sondern nur einer sie in Bewegung bringenden Kraft, welche von außen stammte.

An dieser Stelle erfolgt bei Kant und Jaspers ein Übersprung von der Welt der Natur zur Welt des menschlichen Geistes.

Alle Gegenstände der Natur sind mir als mir gegenüber äußere Gegenstände gegeben. Die Erfahrung meiner selbst ist mir jedoch als eine Erfahrung der Gegenstandslosigkeit gegeben, welche niemals für einen Blick von außen zugänglich sein wird. Die Erfahrung meines Daseins stellt also lediglich in ihrer Art die einzige und ausschließlich richtige Erfahrung des Lebens dar. Die Feststellung, dass es die Existenz von so etwas wie dem Willen gibt, ist dann vollkommend richtig, wenn sie sich nur auf die Erfahrung ,ich will“ bezieht. Das Sprechen davon, dass andere lebende Organismen etwas wollen, oder sogar andere Leute, ist nur ein Bezug auf eine gewisse Analogie gültig. 
Die Wirklichkeit meiner Erfahrung meiner eigenen Gegenwärtigkeit und der Erfahrung eines beliebigen, durch den äußeren Sinn wahrgenommenen Gegenstandes, sind im wesentlichen zwei unterschiedliche Wirklichkeiten für Kant, wie auch für Jaspers. Die Psychologie als Wissen über den Menschen versucht eine Wissenschaft zu sein. In Konsequenz dessen, so Jaspers, ist sie bemüht, sich auf das zu beziehen, worauf sich die Wissenschaften beziehen - also auf die externe Erfahrung; denn nur solch eine Erfahrung kann als eine objektive in diesem Sinne anerkannt werden, da sie mehr als einem Beobachter gegeben ist. Die Wissenschaft kann sich nicht mit einer Erfahrung beschäftigen, welche nur einer Person gegeben ist, indem die Psychologie sich das wissenschaftliche Paradigma stellt, wie man äußere Gegenstände untersucht. Vor allem der Behaviorismus versucht, die Phänomene zu untersuchen, welche die Psyche regieren. Im Endeffekt widerspricht sie sich selbst, denn die Psyche ist uns jedes Mal ausschließlich in einer persönlichen inneren Erfahrung gegeben.

Kant verstand das sehr gut, indem er meinte, dass die Psychologie nie den Status einer vollberechtigten Disziplin der Naturwissenschaft erreichen werde. Anstatt ihrer schlug er das Gebiet, das als Anthropologie in pragmatische Hinsicht bezeichnet wird, vor. Jaspers folgte seiner Spur, obwohl er die Bezeichnung der Anthropologie nicht benutzte. Er meinte, dass diese Bezeichnung so übermäßig oft gebraucht wurde, dass sie letztendlich abgenutzt wurde.

Er unterstrich jedoch vor allem, dass der Übergang von der Wirklichkeit der Natur zur Wirklichkeit des Geistes einen ungleich größeren Sprung bedeute, als der Übergang von der Welt der unbelebten zur belebten Natur. In den heutigen Zeiten, erscheint auf dem Müllplatz der wahren kognitiven Wissenschaften ein Phänomen der Pseudo-Wissenschaft, welche durch Feynman als cargo cult bezeichnet wird. Wissenschaftler, die sie vertreten, beziehen sich auf die Ergebnisse der neurophysiologischen Untersuchungen, von denen sie ein sekundäres und oberflächliches Wissen besitzen. Sie betreiben eine spezifische Disziplin, welche ich als „Kult des Gehirnes“ bezeichnen ließe. Das beiläufig gehörte Wissen aus dem Bereich der Neurophysiologie verbinden sie mit der primitiven Metaphysik, deren Rolle der einfachen Substanz eigentlich vollständig das Gehirn erfüllt. Sie wiederholen die These: alle Denkprozesse entstehen als Effekt neuronaler Prozesse, die im Gehirn vorgehen, wie eine Mantra. Solch eine Tätigkeit, wird entgegen der kognitiven Wissenschaften als „Kognitivismus“ bezeichnet und nimmt heute beunruhigende Ausmaße in Polen an. 
In dem angeführten vierten Kapitel des ersten Bandes der Philosophie wird solch eine Tätigkeit durch Jaspers als Positivismus bezeichnet, die unter anderem als ein Postulat erscheinen kann, dass jegliche Denkprozesse ein Effekt der Aktivität der Materie sind, worauf zahlreiche Korrelate hindeuten.

Das Gegenstück der Positivisten sind die Idealisten, welche verkünden, dass alles, was wir als Materie bezeichnen uns ausschließlich als Denkinhalt gegeben ist. Somit sollte dem Denken die Bezeichnung der ursprünglichen Substanz zustehen, denn die Materie ist nur die Folge von ihr.

Aus der Philosophie von Kant ergibt sich klar, dass alle Typen dieser Auseinandersetzung an Streitigkeiten zweier Gentlemen um die Farbe von Schnee erinnern, von denen der eine eine grüne und der andere eine rote Brille auf hat. Die Tatsache, dass der Denkprozess spontan aus der unbelebten Substanz entstanden ist, ist nie in der Wissenschaftsgeschichte beobachtet worden, ähnlich wie die Tatsache, dass aus solch einer Substanz Leben entstanden ist. Ebenso wie nie beobachtet worden ist, dass es gelungen ist mit einer biologischen unvernünftigen Person anhand der Kategorie von Anzahl, Qualität, Beziehung und Modalität Kontakt aufzunehmen. Jegliche biologischen Evolutionstheorien betreffen ausschließlich die Verbindungen von empirischen Tatsachen, die eines Charakters a posteriori sind. Wenn es also um die Redlichkeit geht, so ist die Hypothese, dass der Denkprozess ein spontanes Produkt der Materie darstellt, nicht glaubhafter als die Hypothese, dass erst das Phänomen des Lebens und dann das des Denkens infolge der Einwirkung einer uns unbekannten Ursache auf der Welt, oder gar gegenüber der Außenwelt verheimlichten Ursache entstanden sind.

Ich bin der Ansicht, dass bei allen bisher präsentierten Motiven Jaspers den Spuren Kants folgt. Nun würde ich gerne zu den Bereichen der Philosophie übergehen, bei denen ich der Meinung bin, dass sie Kant ergänzen.

Indem man sein eigener Geist ist, also eine Erfahrung seiner Gegenstandslosigkeit hat, welche mir durch den äußeren Sinn zugänglich ist, glaube ich stark daran, dass hinter der Fassade eines Gegenstandes, den für mich ein anderer Mensch darstellt, die innere Erfahrung des Ich-seins versteckt ist, analogisch zu der meinen. Solch eine Erfahrung, dass man jemand anders ist, wird mir niemals direkt gegeben sein. Doch mit dem Geist eines zweiten Menschen kann ich anhand der existenziellen Kommunikation Kontakt aufnehmen. Wie erfolgt dieser?

Um es dazu kommen zu lassen, müssen wir uns im großen Masse von dieser Art an Kommunikation befreien, welche unter Einfluss der auf der Welt stattfindenden Richtigkeiten erfolgt. Im Alltag bin ich fast immer ein „etwas“, 
was einen Vorhang meines „Wer-seins“ darstellt. Mit anderen Menschen kommuniziere ich entsprechend der Funktion, welche ich in der Gesellschaft einnehme. Sich der Terminologie Kants bedienend, ist ein zweiter Mensch dann kein Ziel an sich selbst für mich, aber ein Mittel zum Erreichen meiner eigenen Ziele. Darüber hinaus werde ich ständig durch die Furcht gelenkt, die mit meinem Kampf um das Überleben verbunden ist. Ich habe Angst davor, dass jegliches Öffnen meines Inneren vor anderen bedeuten kann, dass ich einer Gefahr ausgesetzt werde. Die existenzielle Kommunikation erziele ich dann, wenn ich in der Lage bin, diese Bestrebungen und Ängste zu überwinden. Jemand, mit dem ich dann kommuniziere, steht mir dann sehr nahe und ich habe keine Angst mich vor ihm zu öffnen.

Die existenzielle Kommunikation bedeutet jedoch kein künstliches Einverständnis, das auf dem Vermeiden von Konflikten um jeden Preis beruht. Solch ein bedingungsloses Einverständnis, welches um jeden Preis erhalten wird, ist eher für die formelle Kommunikation charakteristisch, die z.B. mit dem Erfüllen einer gesellschaftlichen Funktion verbunden ist. Die in einer existenziellen Kommunikation verbleibenden, sich nahen Personen sollten Konflikte miteinander austragen. Darüber hinaus, Jaspers meint, sollten sie miteinander kämpfen. Aber um was wird dann gekämpft? Das ist vor allem kein Kampf, welcher sich aus der Furcht ums Überleben ergibt, oder um eine gesellschaftliche Position, was übrigens eines und das gleiche bedeutet. Bei der existenziellen Kommunikation kann ich nur mit jemandem verbleiben, dem ich vollständig vertraue und vor dem ich mich nicht fürchte. Der Kampf ergibt sich aus der Tatsache, dass jeder von uns bei jeder Angelegenheit nur über eine teilweise Wahrheit verfügt. Indem man sich Argumente vorbringt, kämpfen wir um das Erreichen solch einer Wahrheit, die wir beide akzeptieren können. Letztendlich wird unsere Gemeinschaft zum Ziel dieses Kampfes. Das ist also ein Kampf um die Liebe.

Was bedeutet denn hier das Wort Liebe? Mit Sicherheit, meint Jaspers, nimmt sie bei der Faszination zweier Personen an sich keine Form der Psychose an. Jeder Mensch ist nur ein Mensch, das Wahrnehmen einer Gottheit in ihm ist nur ein ästhetischer Anschein. Die von der Welt gelöste Liebe, eine solchen wie die von Romeo und Julia, war noch keine wahre Liebe. Damit sie zu solch einer wird, muss die gegenseitige Faszination zu einer gemeinsamen Besorgnis beider Seiten um ein gemeinsames Ziel auf der Welt übergehen im Falle von Eheleuten z.B. um die Familie und die Kinder, im Falle von Freunden, um die Welt, die sie gemeinsam lieben, im Falle von Wissenschaftlern (z.B. eines Meisters und Schülers), um ein gemeinsam realisiertes Ziel an 
Untersuchungen. Die Liebe kann also ohne das Verlieben erfolgen, oder ohne eine blinde Faszination an dem, den man liebt. Wenn sie sich jedoch nur an solch eine Faszination anlehnen muss, dann muss das so enden, wie die Liebe von Wroński und Anna Karenina: indem die erotische Faszination übergangen wird, besaßen sie nichts anderes, was sie miteinander verbinden könnte. Sie waren nicht einmal zu solch einer elementaren Sorge, wie die Sorge um das eigene Kind, in der Lage.

Aus der Gemeinschaft zweier im Akt der Kommunikation verbundener Geister entsteht also eine „Wir"-Wirklichkeit, die etwas anderes als die Wirklichkeiten „du“ und „ich“ sind, jede einzeln, aber sie ist auch etwas anderes, als irgendeine Summierung von „ich“ und „du“. Jene Wir-Wirklichkeit funktioniert nach dem folgenden Verlauf. Stellen wir uns vor, dass Hans in einer existenziellen Kommunikation mit Werner verbleibt, gleichsam hingegen mit Helga. Hans, der in solch eine Kommunikation mit Werner eingeht, ist natürlich ein anderer Hans als der, welcher mit Helga kommuniziert. Solch einen Hans, welcher für Werner ist, würde es also nicht geben, würde es Werner nicht geben, ähnlich kann Hans so einer wie er für Helga ist, nur dank Helga sein. Sowohl der eine wie der andere Hans könnte er nicht sein, indem er noch eine Kommunikation mit einem vierten führte, oder auch indem es zu keiner Kommunikation mit irgendjemanden kommt.

Solch eine Gemeinschaft der Geister bei der existenziaellen Kommunikation ist also eine Gemeinschaft, in der eine „wir"-Wirklichkeit geboren wird, die sich nur von der „ich“- und „du“-Wirklichkeit selbst unterscheidet. Die Anzahl der einzelnen Wirklichkeiten zweier, geht hier in die Qualität einer „Wir"-Wirklichkeit über, obwohl sie sowohl ohne „ich“ wie auch ohne „du“ nicht existieren kann, sie ist gänzlich von „,ich“ wie auch „du“ different. Solch eine Wirklichkeit neuer Qualität bildet Hans bei der existenziellen Kommunikation mit Werner, eine zweite, gänzlich andere mit Helga. Solche autonomischen „wir"-Wirklichkeiten kann er so viele gemeinsam mit so vielen erschaffen, mit wie vielen Menschen es ihm gelingt eine existenzielle Kommunikation zu erzielen. Den Geist eines einzelnen Hans Jaspers bestimmt er auch durch die Bezeichnung „Selbst“. Das ist natürlich ein Terminus, der für Hegel charakteristisch ist, dort wo mit ihm eine Einheit als atomistischer Bestandteil der Wirklichkeit des Geistes bezeichnet wird. Doch die Gemeinschaft zweier Geister im Akt der existenziellen Kommunikation ist weiterhin kein einheitlicher Geist mit der Nummer drei, denn sie ist kein Gegenstand. Sie ist eben eine Formation des Geistes. 
Der psychische Prozess, der in dem zuvor beschriebenen äußeren Empfinden erfolgt, ist mit Sicherheit jedem bekannt, der zumindest einmal im Leben einen wahren besten Freund hatte. Ich bin mir sicher, dass solch eine äußere Erfahrung von „uns“ für die Mehrheit von uns gleichsam selbstverständlich ist, wie die äußere Erfahrung des Anblickes von dem Brandenburger Tor. Hegel übersah sie jedoch. Er beschäftigte sich nicht allzu viel mit dem alleinigen Selbst, aber mit der Art, wie es noch weniger zum Bestandteil der Formation des Geistes wird. Und wenn es schon dazu kommt, dann bezog er sich, indem er dieses Phänomen zu erklären versuchte, auf nicht besonders zutreffende Lösungen. Die Habsucht des Geistes, der Konflikt zwischen dem Herren und dem Sklaven, das unglückliche Bewusstsein - all diese Erklärungen deuten darauf hin, dass der Geist zum Betrug greifen kann, zum Versprechen eines Preises oder eines Zwanges, wenn eine Einheit sich selbst dem Prozess des Werdens zu einem Geist abgeben möchte. Das Glück hingegen oder das Unglück einer Einheit ist somit hier nicht mehr von Bedeutung, wie in dieser Perspektive zutreffend die zuvor angeführte Ironie Kierkegaards lautet: wozu einen Palast bauen, wenn der Eigentümer in der sich neben ihm befindlichen Hundehütte wohnen muss?

Und doch, wofür Popper zum Beispiel Hegel die Schuld gibt, war das zwanzigste Jahrhundert eine Ära großer Ideologen, welche, indem sie großartige Gesellschaften versprachen, sie den Einheiten befahlen, in Hundehütten zu wohnen. Eine solcher Einheiten war ich selbst, denn meine Kindheit, Jugend und überhaupt mehr als die Hälfte meines Lebens habe ich in einem kommunistischen Staat gelebt.

Jaspers vermied in diesem Punkt die Kurzsichtigkeit Hegels, was hervorragend seine wundervolle Phrase ausdrückt:

„Wer nur die Menschheit liebt, liebt gar nicht, wohl aber, wer diesen bestimmten Menschen liebt".

Indem die Einheit geliebt wird und man mit ihr in eine existenzielle Kommunikation eintritt, bilden wir nämlich die kleinste Formation des Geistes, also die kleinste Formation der Menschlichkeit. Je mehr einzelne Personen solche existenziellen Gemeinschaften bilden - das müssen jedoch keine aus ausschließlich zwei Personen bestehenden Gemeinschaften sein - desto stärker wird die Menschheit selbst.

Es gibt das interessante Phänomen, dass zu unserer Zeit, als der Gesellschaft ihr autonomische Sein entzogen wird, sich die existenzielle Kom-

\footnotetext{
${ }^{4}$ K. Jaspers, Philosophie, S. 14.
} 
munikation besonders entwickelt. In Zeiten, in denen der kommunistische Terror tobte, und der Einkauf eines Würfels an Butter in einem Geschäft als ein glückliches Ereignis angesehen wurde, erlebte das Phänomen zwischenmenschlicher existenzieller Kommunikation das höchste Entwicklungsniveau. Zu seinem Symbol ist die Bewegung "Solidarnośćc geworden, welche mit nichts anderem in der Weltgeschichte vergleichbar ist.

Auf die Frage, wieso „Solidarność” heute eine Geschichte ist, fällt es nicht schwer, eine Antwort zu finden. So wie der Organismus gewisse Antikörper in großer Anzahl produziert, nur wenn er in einem kritischen Zustand ist, so ist die Situation, in der ich mit jedem zufälligen Menschen einfach eine existenzielle Kommunikation dank Gott erziele, keine alltägliche Situation. Zu normalen Zeiten habe ich eine starke Bindung mit den besten Freunden, der Familie, den Studenten, also allen, mit denen mich eine existenzielle Sorge um eine Person und eine Angelegenheit verbindet. Das ist heute nicht, dank Gott, die Sorge um für die Menschheit bahnbrechende Fragen, oder sogar um mein Land. Ich denke, dass niemand von den geehrten Freunden die sensationellen Meldungen von den hysterischen Politikern und den Journalisten nicht ernst nimmt, dass die Demokratie in Polen ernsthaft gefährdet sei. Heute kümmerten wir uns mit der Gruppe meiner Studenten aus meinem Seminar um die Übersetzung von Jaspers Philosophie in die polnische Sprache. Unsere Arbeit weckt eine allgemeine Sympathie, aber zu ihrem Thema werde ich nicht mehr eine existenzielle Kommunikation mit meinem alten Vater, oder der Cousine einer Putzfrau aufnehmen. Ich erinnere mich jedoch an die Zeiten, als wir uns während des gleichen Seminars um Krystyna sorgten, die für das Besitzen illegaler Flugschriften verhaftet worden ist. Wir stellten uns die Frage: wer hat sie denunziert? Indem wir über ihre Inhaftnahme sprachen, konnte man die existenzielle Kommunikation beinahe mit jedem zufällig in Polen getroffenen Menschen erreichen.

Das sensationelle Interview Heideggers machte schon immer den Eindruck eines Kritsches auf mich. Wir lesen dort über schlechte Zeiten, darüber, dass die Philosophie endlich durch die Kybernetik ersetzt wurde, denn wie sollte es anders sein: nur Gott könnte uns noch retten. Wir hören unaufhörlich von der Krise der Philosophie, dem Anschlag der Kulturen u. ä.

Gab es jedoch in der Geschichte der Philosophie eine Epoche, in der es keine Krise gab? Was für eine Krise gab es zur Zeiten von Sokrates, Descartes, Kant, Husserl!

In der ganzen Schwarzseherei kann nur ein Argument wahr sein: dass die Menschheit mit Sicherheit irgendwann zu existieren aufhört. Zuvor jedoch 
wird jeder von uns sterben, dann geht die Sonne aus und das Universum wird ausbrennen.

Es geht mir aber nicht um solch eine Schwarzmalerei, sondern um das, was Heidegger feststellte, indem er sagte, dass die Philosophie schon beendet ist und höchstens etwas geboren werden kann, was er als zweites Denken bezeichnen würde. Sie endete, denn die Menschen möchten nicht mehr so denken, wie es sich Heidegger ausgemalt hat.

Im Oktober des Jahres 1973 habe ich meinen ersten Satz von Jaspers ins Polnische übersetzt. Er lautet so: „Wir wagen zu behaupten: Philosophie kann nicht aufhören, solange Menschen leben”.

Die großen philosophischen Systeme entstehen zu gewissen Zeiten, zu anderen nicht. Wenn sie nicht entstehen, dann können die alten Systeme durch die ganzen Jahrhunderte und Jahrtausende hindurch genutzt werden. Doch die Werke großer Philosophen stellen für Jaspers nur die Ruine der Größe ihrer Schöpfer dar. Die wahre Philosophie ist jedoch kein Set an Systemen sondern ein Philosophieren. Das Philosophieren hingegen beruht auf jeglicher Suche nach der Wahrheit durch die Einheiten in der Aktion der existenziellen Kommunikation. Ohne der existenziellen Kommunikation, als einer Quelle sowie der letzten Instanz wäre keine Kommunikation möglich. Damit die Philosophie stirbt, müsste es zu einem Zustand kommen, in dem alle menschlichen Gemeinschaften zum Erliegen kommen würden, und nicht nur diese, an die wir uns gewöhnt haben. Mit anderen Worten müsste es zu einer Degeneration des Menschen als einem gesellschaftlichen Wesen kommen, dann zurück zur Tierheit, also müsste es faktisch zu einer Umkehrung des Entwicklungsprozesses kommen. Danach sieht es aber nicht aus. Die existenzielle Kommunikation zwischen den Menschen besteht weiterhin und niemand kann vorhersehen, wie und wann das fruchtet. Sogar Heidegger selbst war von dem Vergehen seiner Welt entsetzt.

\section{Literatur}

Jaspers K., Philosophie, Berlin-Göttingen-Heidelberg 1948.

Kant I., Gesammelte Schriften. Hg.: Bde. 1-22 Preussische Akademie der Wissenschaften, Bd. 23 Deutsche Akademie der Wissenschaften zu Berlin, ab Bd. 24 Akademie der Wissenschaften zu Göttingen, Berlin 1900 ff. 


\begin{abstract}
Kant and Jaspers

Hannah Arendt called Jaspers "the Kant of the $20^{\text {th }}$ century". This is an apt term, though Jaspers was certainly neither a Kantian nor a Neo-Kantian. Kantianism is connected with philosophizing for which epistemology was the most important, especially in the sphere of speculative reason, and the main goal of that philosophy was to create the tools necessary for the proper development of science. In Jaspers' opinion, neo-Kantianism, even if it dealt with social problems, it focused only on the first of the three Kantian questions that exhaust problems of philosophy („What can I know?”, „What should I do?", „What can I expect?”). These issues are, among others, discussed in this article with a focus on a search for a justification for the thesis put forward by Hannah Arendt.
\end{abstract}

Key words: Kant, Jaspers, Kantianism, neo-Kantianism, existentialism 